\title{
The effect of BPIFA1/SPLUNC1 genetic variation on its expression and function in asthmatic airway epithelium
}

\author{
Niccolette Schaefer, ${ }^{1}$ Xingnan Li, ${ }^{2}$ Max A. Seibold, ${ }^{1}$ Nizar N. Jarjour, ${ }^{3}$ Loren C. Denlinger, ${ }^{3}$ \\ Mario Castro, ${ }^{4}$ Andrea M. Coverstone, ${ }^{4}$ W. Gerald Teague, ${ }^{5}$ Jonathan Boomer, ${ }^{4}$ Eugene R. Bleecker, ${ }^{2}$ \\ Deborah A. Meyers, ${ }^{2}$ Wendy C. Moore, ${ }^{6}$ Gregory A. Hawkins, ${ }^{6}$ John Fahy, ${ }^{7}$ Brenda R. Phillips, ${ }^{7}$ \\ David T. Mauger, ${ }^{8}$ Azzeddine Dakhama, ${ }^{1}$ Shaan Gellatly, ${ }^{1}$ Nicole Pavelka, ${ }^{1}$ Reena Berman, ${ }^{1}$ \\ Y. Peter Di, ${ }^{9}$ Sally E. Wenzel, ${ }^{9}$ and Hong Wei Chu ${ }^{1}$ \\ ${ }^{1}$ National Jewish Health, Denver, Colorado, USA. ${ }^{2}$ University of Arizona, Tucson, Arizona, USA. ${ }^{3}$ University of Wisconsin- \\ Madison, Madison, Wisconsin, USA. ${ }^{4}$ Washington University in St. Louis, St. Louis, Missouri, USA. ${ }^{5}$ University of Virginia, \\ Charlottesville, Virginia, USA. 'Wake Forest University, Winston-Salem, North Carolina, USA. UCSF, San Francisco, \\ California, USA. ${ }^{8}$ Pennsylvania State University, Centre County, Pennsylvania, USA. ${ }^{9}$ University of Pittsburgh, Pittsburgh, \\ Pennsylvania, USA
}

Bacterial permeability family member A1 (BPIFA1), also known as short palate, lung, and nasal epithelium clone 1 (SPLUNC1), is a protein involved in the antiinflammatory response. The goal of this study was to determine whether BPIFA1 expression in asthmatic airways is regulated by genetic variations, altering epithelial responses to type 2 cytokines (e.g., IL-13). Nasal epithelial cells from patients with mild to severe asthma were collected from the National Heart, Lung, and Blood Institute Severe Asthma Research Program centers, genotyped for rs750064, and measured for BPIFA1. To determine the function of rs750064, cells were cultured at air-liquid interface and treated with IL-13 with or without recombinant human BPIFA1 (rhBPIFA1). Noncultured nasal cells with the rs750064 CC genotype had significantly less BPIFA1 mRNA expression than the CT and TT genotypes. Cultured CC versus CT and TT cells without stimulation maintained less BPIFA1 expression. With IL-13 treatment, CC genotype cells secreted more eotaxin-3 than CT and TT genotype cells. Also, rhBPIFA1 reduced IL-13-mediated eotaxin-3. BPIFA1 mRNA levels negatively correlated with serum IgE and fractional exhaled nitric oxide. Baseline FEV1\% levels were lower in the asthma patients with the CC genotype $(n=1,016)$. Our data suggest that less BPIFA1 in asthma patients with the CC allele may predispose them to greater eosinophilic inflammation, which could be attenuated by rhBPIFA1 protein therapy.

Authorship note: NS and XL are cofirst authors.

Conflict of interest: The authors have declared that no conflict of interest exists.

Copyright: (c) 2019 American Society for Clinical Investigation

Submitted: January 7, 2019

Accepted: March 14, 2019

Published: April 18, 2019

Reference information: /Cl Insight. 2019;4(8):e127237. https://doi. org/10.1172/jci.insight.127237.

\section{Introduction}

Asthma is a heterogeneous disease that has at least 2 broad endotypes: type 2 high and type 2 low. Patients with type 2-high asthma have higher levels of type 2 cytokines, such as IL-13, IL-4, and IL-5, which are associated with higher levels of IgE, eosinophils, and periostin (1-3). How airway innate immunity regulates type 2 inflammation remains a topic of interest. Because airway epithelium serves as a critical first line of host innate immunity, previous research has focused on its role in type 2 inflammation. Bacterial permeability family member A1 (BPIFA1), also known as short palate, lung, and nasal epithelium clone 1 (SPLUNC1), is a secretory protein primarily produced by airway (nasal, bronchial) epithelium (4-7). BPIFA1 has been reported to exert antiinflammatory properties during microbial infections and Toll-like receptor agonist stimulation (8-13). We and others (14-16) have found that IL-13 and allergen challenges significantly reduce airway epithelial expression of BPIFA1, which in turn further exaggerates type 2 inflammation (16).

The impact of genetic variation on BPIFA1 expression in asthmatic airway epithelium has not been investigated. An early study in a Chinese population (17) suggests that single nucleotide polymorphisms (SNPs) of BPIFA1 (e.g., the rs750064 CC genotype) were associated with increased susceptibility to nasopharyngeal carcinoma. A recent study in cystic fibrosis (CF) patients' lungs (18) demonstrated that the minor allele $\mathrm{G}$ of rs1078761 located in exon 3 of long PLUNC1 (LPLUNC1) reduced lung function and 
Table 1. Demographic information of adult subjects with asthma from the SARP3 cohort

\begin{tabular}{|c|c|c|c|}
\hline rs750064 & CC & СT & TT \\
\hline $\mathrm{N}$ & 17 & 24 & 8 \\
\hline Race (White/African American) & $10 / 7$ & $23 / 1$ & $8 / 0$ \\
\hline Age (yrs) & $45.0 \pm 13.6$ & $50.5 \pm 16.8$ & $45.7 \pm 14.9$ \\
\hline Age of asthma onset (yrs) & $17.5 \pm 15.5$ & $18.2 \pm 16.8$ & $16.7 \pm 14.2$ \\
\hline Asthma severity & 8 mild/moderate, 9 severe & 8 mild/moderate, 16 severe & 2 mild/moderate, 6 severe \\
\hline FEV1/FVC & $0.68 \pm 0.11$ & $0.73 \pm 0.10$ & $0.73 \pm 0.11$ \\
\hline FEV1 (\%) & $83.9 \pm 17.9$ & $92.8 \pm 22.6$ & $87.3 \pm 15.3$ \\
\hline$\%$ on ICS & 64.7 & 66.7 & 62.5 \\
\hline$\%$ on other CS & 17.6 & 58.3 & 12.5 \\
\hline BMI & $30.2 \pm 7.4$ & $31.4 \pm 7.9$ & $31.9 \pm 6.9$ \\
\hline
\end{tabular}

FVC, forced vital capacity; ICS, inhaled corticosteroids; other CS, oral/injected corticosteroids. BMI, body mass index $\left(\mathrm{kg} / \mathrm{m}^{2}\right)$.

expression of LPLUNC1 as well as BPIFA1. However, there is a minimal effect of rs750064 on lung tissue BPIFA1 expression in CF. Of note, these studies did not specifically examine BPIFA1 in airway epithelial cells, the major source of BPIFA1.

In the current study, we hypothesized that BPIFA1 expression is partly controlled by genetic variations, which affect the epithelial response to type 2 cytokines. To test this hypothesis, we obtained nasal brushing samples from subjects with asthma participating in the National Heart, Lung, and Blood Institutesponsored (NHLBI-sponsored) Severe Asthma Research Program 3 (SARP3). We genotyped rs750064, measured BPIFA1 expression by nasal epithelial cells, and performed air-liquid interface culture of nasal epithelial cells carrying different alleles of rs750064 to determine the effect of BPIFA1 genetic variation on IL-13-mediated production of proeosinophilic mediator eotaxin-3 (CCL26). We also tested the therapeutic effect of recombinant human BPIFA1 protein on IL-13-mediated eotaxin-3. Additionally, we correlated BPIFA1 expression with type 2 markers and pulmonary function in patients with asthma.

\section{Results}

Unlike the invasive nature of bronchoscopy used to obtain the brushed bronchial epithelial cells, nasal brushing is considered minimally invasive and can be done in adults and children. Moreover, nasal epithelial cells were shown to demonstrate similar gene expression pattern to bronchial epithelial cells (19). To determine whether BPIFA1 expression in nasal epithelial cells may predict that in bronchial epithelial cells, in a pilot study, we analyzed RNA sequencing data of brushed nasal and bronchial epithelial cells (time of sampling, noncultured) obtained from the same healthy subjects $(n=7)$ and stable subjects with asthma $(n=9)$ (ages, $19-52$ years). Supplemental Figure 1 (supplemental material available online with this article; https://doi.org/10.1172/jci.insight.127237DS1) demonstrates a strong positive correlation between BPIFA1 mRNA expression in nasal and bronchial epithelial cells. Thus, nasal brushing may serve as a robust and minimally invasive approach to study BPIFA1 expression in asthma.

Variant rs750064 affects BPIFA1 expression in brushed asthmatic nasal epithelial cells BPIFA1 mRNA expression levels in brushed (noncultured) nasal epithelial cells were compared in 74 adult and pediatric subjects with asthma (49 adult, Table 1; 25 pediatric, Table 2). As shown in Figure 1A, BPIFA1 mRNA levels in nasal epithelial cells expressing the CC genotype $(n=27)$ were about 30 -fold lower $(P$ $<0.0001)$ than those expressing the CT $(n=34)$ or TT $(n=13)$ genotype. Interestingly, BPIFA1 mRNA levels were similar between the cells expressing the $\mathrm{CT}$ or TT genotype, suggesting the $\mathrm{T}$ allele as the dominant allele and the $\mathrm{C}$ allele as the recessive allele. BPIFA1 mRNA expression levels were similar among adult and pediatric subjects with asthma, as well as in those with severe versus nonsevere asthma.

To test whether differential expression of BPIFA1 by genotypes in noncultured, brushed cells can be maintained in cultured cells, BPIFA1 mRNA expression was analyzed in expanded nasal epithelial cells under the submerged culture without any treatment. Once again, the CC genotype showed significantly less 
Table 2. Demographic information of pediatric subjects with asthma from the SARP3 cohort

\begin{tabular}{lccc}
\hline rs750064 & CC & CT & TT \\
N & 10 & 10 & 5 \\
Race (White/African American) & $2 / 9$ & $9 / 1$ & $5 / 0$ \\
Sex (male/female) & $6 / 5$ & $5 / 5$ & $3 / 2$ \\
Age (yrs) & $12.6 \pm 1.5$ & $12.5 \pm 2.1$ & $13.6 \pm 2.0$ \\
Age of asthma onset (yrs) & $1.8 \pm 1.9$ & $2.0 \pm 2.4$ & $3.3 \pm 2.8$ \\
Asthma severity & 6 mild/moderate, 4 severe & 6 mild/moderate, 4 severe & 4 mild/moderate, 1 severe \\
No. of exacerbations & $0.7 \pm 1.3$ & $0.5 \pm 1.0$ & $1.6 \pm 2.1$ \\
(within last 12 months) & $0.76 \pm 0.08$ & $0.80 \pm 0.11$ & $0.72 \pm 0.03$ \\
FEV1/FVC & $108.8 \pm 7.1$ & $101.8 \pm 12.9$ & $105.5 \pm 13.5$ \\
FEV1 (\%) & 80 & 60 & 20 \\
\% on ICS & 50 & 60 & 80 \\
\% on other CS & $20.4 \pm 1.8$ & $22.7 \pm 4.6$ & $25.9 \pm 6.3$
\end{tabular}

FVC, forced vital capacity; ICS, inhaled corticosteroids; other CS, oral/injected corticosteroids; BMI, body mass index $\left(\mathrm{kg} / \mathrm{m}^{2}\right)$.

BPIFA1 mRNA expression than the CT and TT genotypes (Figure 1B). Expression of BPIFA1 mRNA was measured after nasal epithelial cells underwent 16 days of air-liquid interface (ALI) culture (mucociliary differentiation), and again subjects with the CC genotype maintained significantly lower BPIFA1 mRNA expression (Figure 1C).

Functional implication of rs750064 in the cultured human nasal epithelial response to IL-13 treatment

The effect of rs750064 on BPIFA1 protein expression in nasal epithelial cells cultured at the ALI. After 16 days of mucociliary differentiation, BPIFA1 protein levels were significantly lower in the CC genotype than the CT and TT genotypes in the absence of IL-13 (Figure 2). IL-13 significantly reduced BPIFA1 levels in all the genotypes as previously reported (14-16).

The effect of rs 750064 on nasal epithelial proinflammatory responses to IL-13 treatment. Eotaxin-3 in basolateral supernatants of ALI-cultured nasal epithelial cells was measured by ELISA to determine whether rs750064-dependent BPIFA1 expression affects the epithelial proinflammatory response to IL-13. Eotaxin-3 was significantly induced by IL-13 treatment as previously reported $(20,21)$. Cells with the CC genotype were significantly more responsive to IL-13 treatment, because they had higher eotaxin-3 levels than the CT and TT genotypes (Figure 3A).

Recombinant human BPIFA1 protein inhibits the proinflammatory response to IL-13 treatment. The addition of recombinant human BPIFA1 (rhBPIFA1) protein into the apical surface of nasal epithelial cells cultured at the ALI significantly decreased IL-13-induced eotaxin-3 production in cells carrying both CC and CT or TT genotypes, leaving both genotypes with similar eotaxin-3 levels (Figure 3B). The CC genotype showed a 140-fold reduction in eotaxin-3 levels after rhBPIFA1 treatment, compared with a 100-fold reduction in the CT and TT genotypes.

Type 2 inflammation signatures in brushed nasal epithelial cells carrying different genotypes of rs750064

Because BPIFA1 deficiency leads to greater type 2 inflammation in mice challenged with ovalbumin (16), we determined whether nasal cells expressing the CC genotype demonstrated enhanced type 2 signatures, such as periostin and eotaxin (22-24). Periostin mRNA levels tended to be higher in cells expressing the CC genotype than those expressing the CT or TT genotype (Figure 4A) and were negatively correlated with BPIFA1 levels, as shown in Figure 4B. When race was considered, the correlation of SPLUNC1 with periostin mRNA was more significant in the White subjects than African Americans after adjustment for age and sex. Eotaxin-3, an eosinophil chemoattractant, was measured in the noncultured nasal epithelial cells. The cells carrying the CC genotype showed slightly higher median eotaxin-3 mRNA levels $(P=0.09)$ than those carrying the CT and TT genotypes (Figure $4 \mathrm{C})$. 
A
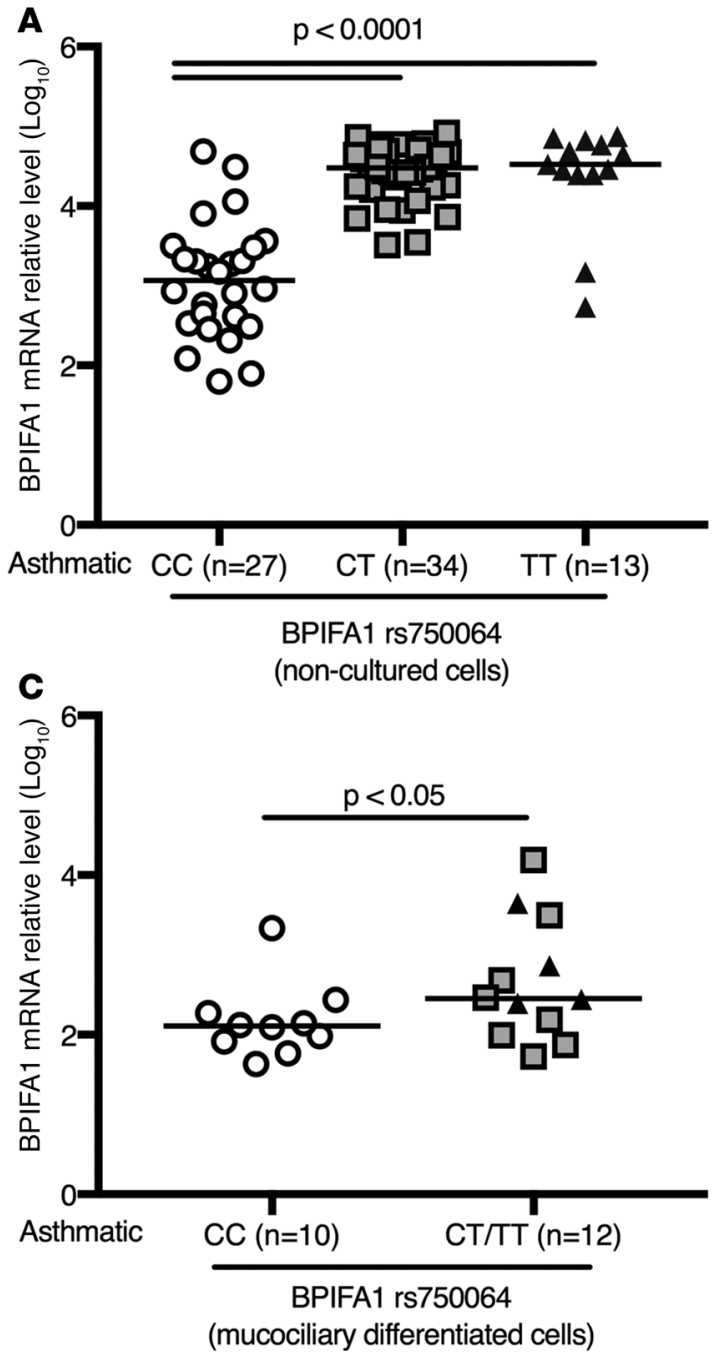

B

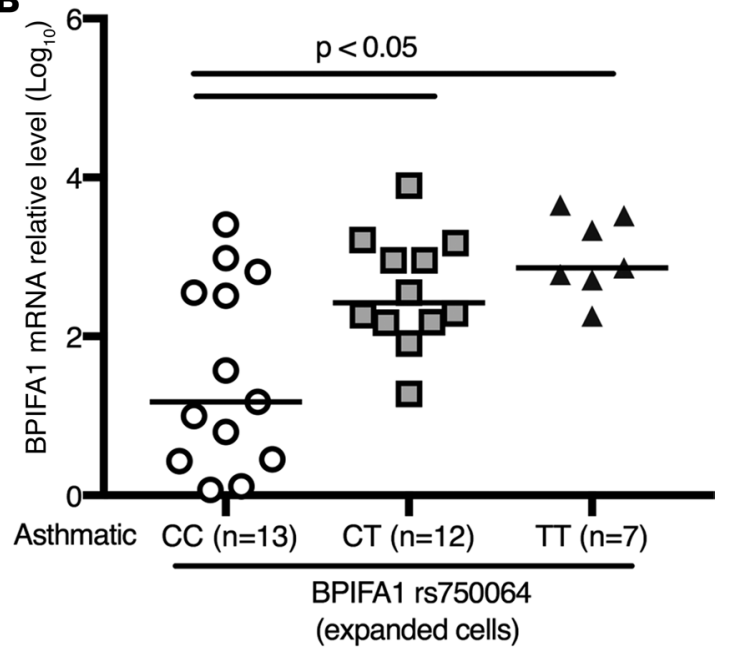

Figure 1. Subjects with the rs750064 CC genotype have significantly less BPIFA1 mRNA expression than subjects with the CT and TT genotypes. BPIFA1 mRNA expression was measured from (A) noncultured nasal epithelial cells of 74 mild to severe subjects with asthma ( $n=49$ adults, $n=25$ children), (B) expanded (submerged culture for 10 days) nasal epithelial cells from 37 asthmatics ( $n=33$ adults, $n=4$ children) without any stimulation, and (C) differentiated (ALI culture for 14 days) mucociliary nasal epithelial cells from subjects with various genotypes (10 CC: 8 White, 2 African American; 8 CT: 8 White, 0 African American; and 4 TT: 4 White, 0 African American) without any stimulation. $P$ values from $\mathbf{A}$ represent a linear regression additive genetic model with adjustments for age, sex, and race; $\mathbf{B}$ represents a Kruskal-Wallis test; and $\mathbf{C}$ represents a Mann-Whitney test. The black line represents the median value.

Correlations of BPIFA1 expression with blood and airway type 2 biomarkers as well as pulmonary function

We examined the relationship of nasal epithelial BPIFA1 expression to blood and airway type 2 biomarkers, such as serum IgE, fractional exhaled nitric oxide (FeNO), and blood eosinophils. BPIFA1 mRNA levels in nasal brushings collected from subjects in the SARP3 study cohort were inversely $(P<0.05)$ correlated to serum IgE (Figure 5A) and FeNO levels (Figure 5B). Because there appeared to be several outliers of serum IgE and FeNO data, we further analyzed the data without these outliers and found that the significance of correlations between BPIFA1 mRNA and serum IgE and FeNO was reduced $(P>0.05)$. Although both serum IgE and FeNO were numerically higher in the $\mathrm{CC}$ group than the $\mathrm{CT}$ and TT groups, the differences were not statistically different. There was no significant correlation between nasal epithelial BPIFA1 expression and number of blood eosinophils in all the subjects expressing different BPIFA1 genotypes $(r=0.06, P=0.62)$. Patients with asthma carrying the CC genotype showed marginally higher levels of eosinophils in induced sputum (Supplemental Figure 2), but not blood (data not shown) samples, than those carrying the CT and TT genotypes.

In 1,016 asthma subjects (non-Hispanic White) enrolled in the SARP1, SARP2, and SARP3 cohorts (Table 3), the rs750064 SNP was significantly associated with baseline FEV1\% levels (nominal $P=0.045$ ) with adjustment for age, sex, and genetic ancestry (principal components 1-5 calculated based on sequencing data), where the CC genotype had significantly lower FEV1\% levels compared with the other 2 genotypes. However, in the population of African Americans with asthma, FEV1\% levels were not significantly different among the rs750064 genotypes (data not shown).

There were no significant associations between rs750064 and the other clinical data, including asthma severity, age of asthma onset, the frequency of asthma exacerbations over the previous 12 months, and BMI. 


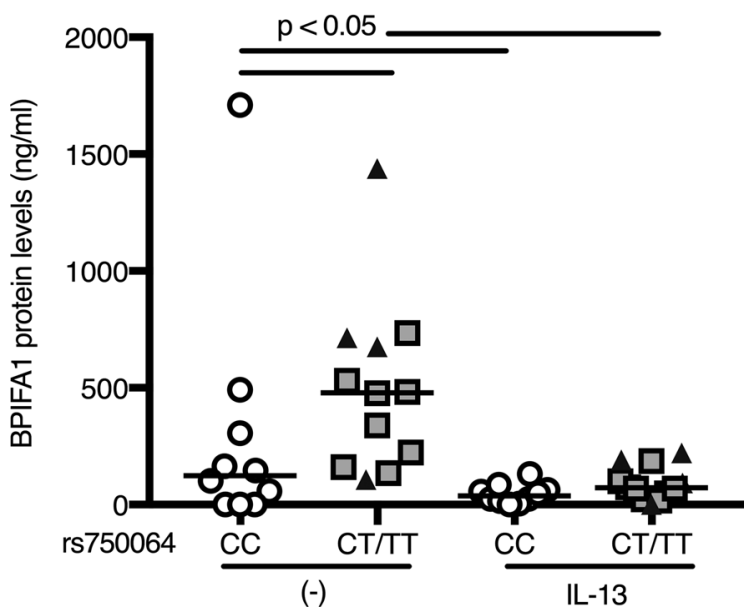

Figure 2. BPIFA1 protein levels remain significantly lower in CC genotypes after $\mathbf{1 6}$ days of ALI culture. Nasal epithelial cells with the rs750064 CC genotype have significantly lower levels of BPIFA1 protein than the CT and TT genotypes. IL-13 treatment significantly reduces BPIFA1 protein. The $P$ values represent a Kruskal-Wallis test. The gray squares and black triangles represent the CT and TT genotype, respectively. The black line represents the median value. $n=10$ CC (8 White, 2 African American); $n=8 \mathrm{CT}$ (8 White, 0 African American) and $n=4$ TT (4 White, 0 African American).

Although no healthy controls were enrolled in the SARP3 cohort for the nasal brushing study, we were able to analyze the correlation of rs750064 with pulmonary function in normal subjects recruited in the SARP1 and SARP2 cohorts. Unlike in the patients with asthma, similar FEV1\% $(P>0.05)$ values were observed in normal, healthy non-Hispanic Whites (Table 4) as well as African Americans (not shown).

\section{Discussion}

Our study shows that BPIFA1 expression in asthmatic nasal epithelium is in part controlled by the genetic variations related to the rs750064 SNP, where the CC genotype has lower BPIFA1 than the CT and TT genotypes. Our potentially novel findings from nasal epithelial cells suggest the strong predictability of rs750064 genotypes in airway BPIFA1 expression. We propose that this genetic variability in airway cell expression of BPIFA1 in subjects with asthma may predispose them to greater eosinophilic inflammation, a feature associated with type 2 inflammation.

Within the BPIFA1 gene, rs750064 is located in the promoter region. Whether rs750064-related BPIFA1 expression is stable from an in vivo to an in vitro setting has not been investigated. Because our preliminary study (Supplemental Figure 1) demonstrated a significantly positive correlation of BPIFA1 mRNA expression in human nasal and bronchial epithelial cells, our data further support the use of nasal brushings as a minimally invasive approach to bronchial brushings in the study of epithelial cell functions related to the BPIFA1 protein. By examining BPIFA1 expression in nasal epithelial cells collected at the time of brushing, and cultured under both undifferentiated (submerged) and differentiated (ALI) conditions, we found that BPIFA1 expression is highly dependent on the rs750064 genotypes, where the CC genotype cells expressed the lowest levels of BPIFA1. Of note, the nasal epithelial cells collected in vivo and cultured at the ALI expressed more BPIFA1 than the undifferentiated nasal epithelial cells. These data further support the concept that secretory cells may be the major producer of BPIFA1, which needs further confirmation using the single-cell RNA sequencing approach. To determine the impact of rs750064 on BPIFA1 expression under a pathological condition, type 2 cytokine IL-13 was added to well-differentiated nasal epithelial cells. Similar to our previous work (and the work of others) (14, 15, 25, 26), IL-13 significantly reduced BPIFA1 expression, suggesting the contribution of both genetic and environmental factors to BPIFA1 expression. However, because of the overwhelmingly inhibitory effect of IL-13 on BPIFA1 expression in vitro, the genetic impact of rs 750064 on BPIFA1 levels was minimal when IL-13 was present. Our selection of the IL-13 dose $(10 \mathrm{ng} / \mathrm{ml})$ is extensively used in the literature $(14,21,27,28)$. Nevertheless, it would be interesting to use lower doses of IL-13 in future studies to determine whether the rs750064 CC genotype affects the sensitivity to the suppressing effect of IL-13 on BPIFA1 in airway epithelial cells. 
A

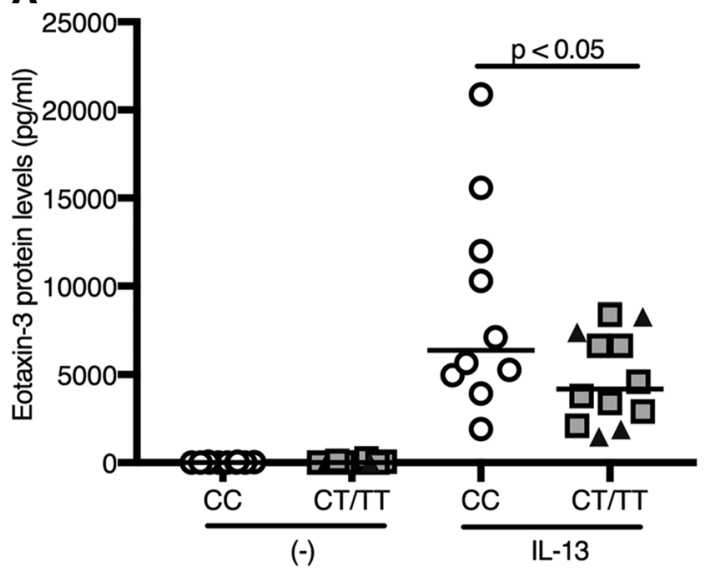

B

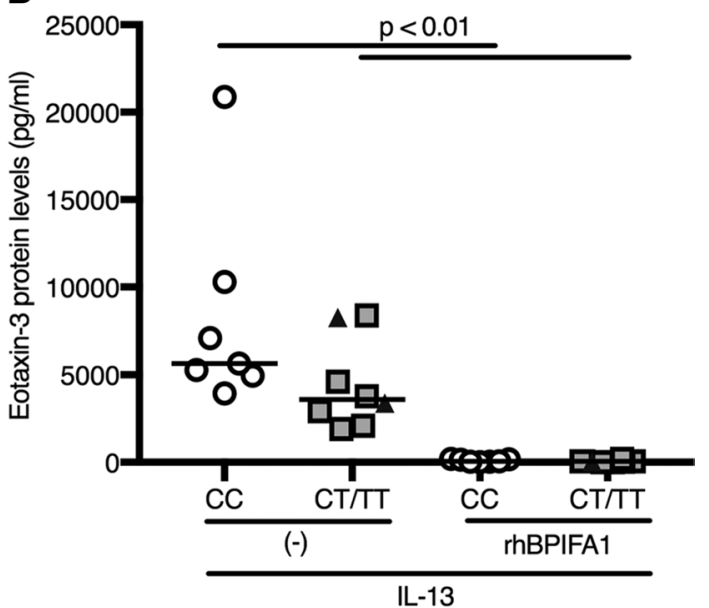

Figure 3. Eotaxin-3 induction by IL-13 is more robust in rs750064 CC genotype cells. (A) In asthmatic nasal epithelial cells cultured at the ALI, IL-13induced eotaxin-3 levels are significantly higher in the rs750064 CC genotype cells than the CT and TT genotype cells ( $n=10$ for CC: 8 White, 2 African American; $n=8$ CT: 8 White, 0 African American; $n=4$ for TT: 4 White, 0 African American). The $P$ value represents the Mann-Whitney test. The black line represents the median value. (B) For all rs750064 genotypes, rhBPIFA1 protein significantly inhibits IL-13-induced eotaxin-3 ( $n=7$ CC: 5 White, 2 African American; $n=6$ CT: 6 White, 0 African American, and $n=2$ TT: 2 White, 0 African American). The $P$ values for indicated comparisons represent a Kruskal-Wallis test. The gray squares and black triangles represent the CT and TT genotypes, respectively. The black line represents the median value.

We found that low BPIFA1 levels modestly predicted the degree of type 2 inflammation reflected by periostin and eotaxin-3 in nasal epithelial cells, serum IgE, and FeNO. The relatively weak correlations of BPIFA1 mRNA and type 2 markers in the current study may be in part explained by the fact that these subjects are in the stable phase of disease. Eosinophils in the blood and sputum have been used as a marker of type 2 inflammation $(1,2,29)$. In our current study, asthmatics carrying the CC genotype had marginally higher levels of eosinophils in induced sputum, but not blood samples, than those carrying the CT and TT genotypes. Once again, this may be due to less active inflammation during the stable phase of the disease. This speculation was supported by the observation that when differentiated nasal epithelial cells were treated with IL-13 to mimic a type 2-high airway, eotaxin-3 was significantly higher in the CC genotype $(P<0.05)$. Moreover, ovalbumin-challenged, BPIFA1-deficient mice demonstrated exaggerated airway eosinophilic inflammation (16). Together, our data suggest that reduced BPIFA1 levels may predispose the airways to more robust eosinophilic inflammation in vivo. However, how BPIFA1 regulates eosinophilic inflammation is unclear. Although we focused on the inhibitory effect of BPIFA1 on eotaxin production, the exact signaling pathways need to be further examined.

Although FEV1\% in the SARP3 subjects expressing the CC genotype tended to be less than the percentage in the other 2 genotypes, it was not statistically significant. However, by using the subjects recruited from the 3 SARP cohorts, we found a significant baseline reduction in FEV1\% levels in non-Hispanic, White patients with asthma carrying the CC genotype. Therefore, rs750064 appears to have a marginal impact on baseline lung function, because BPIFA1 levels did not affect disease severity. This may be in part explained by the fact that the subjects included in this report were stable and not experiencing an acute asthma exacerbation.

There are some limitations to our study. First, the current study did not directly address how rs750064 affects BPIFA1 transcription or translation. Second, there are multiple BPIFA1 SNPs (17), and whether those additional BPIFA1 genetic variations affect its expression and function remains to be determined. Last, because the focus of the SARP3 cohort was on enrolling asthmatics, only nasal epithelial cells from the subjects with asthma were available to our study. Future studies are warranted to recruit normal, healthy subjects to clarify whether rs750064-related differences in BPIFA1 expression and functions are restricted to asthma. Nonetheless, in our pilot study, we measured BPIFA1 mRNA expression of noncultured nasal brushing cells obtained from 15 nonasthmatic subjects who did not have a known lung disease $(n=5)$ or were diagnosed with other lung diseases $(n=10)$, such as sarcoidosis. We found that like the asthmatic subjects, nonasthmatic subjects with the rs750064 CC genotype expressed lower levels of BPIFA1 mRNA than those expressing the other 2 genotypes (Supplemental Figure 3). Thus, a combination of rs750064 and other factors (genetic and environmental) may eventually determine asthma pathobiology. 
A

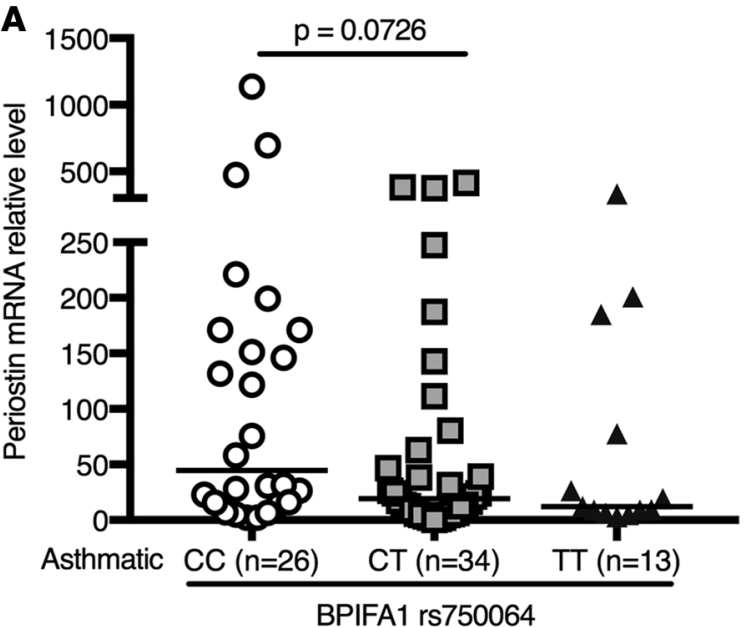

BPIFA1 rs750064
B

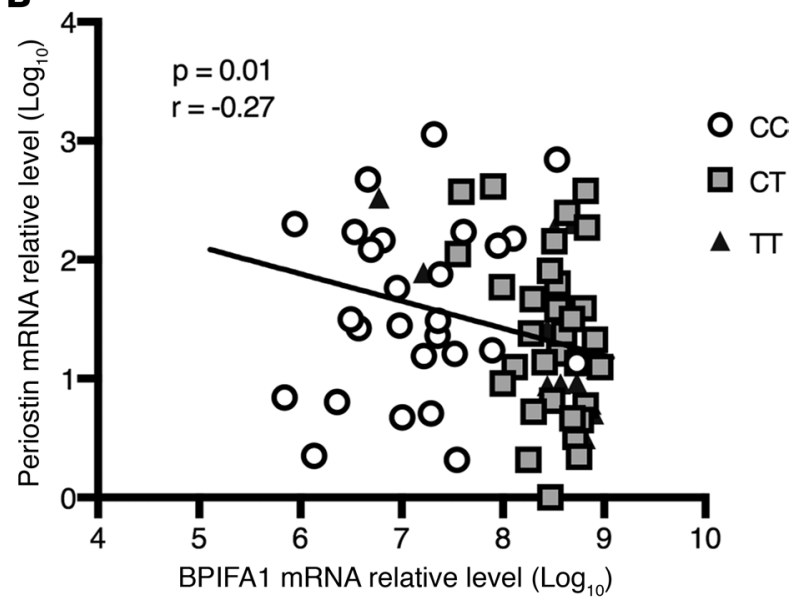

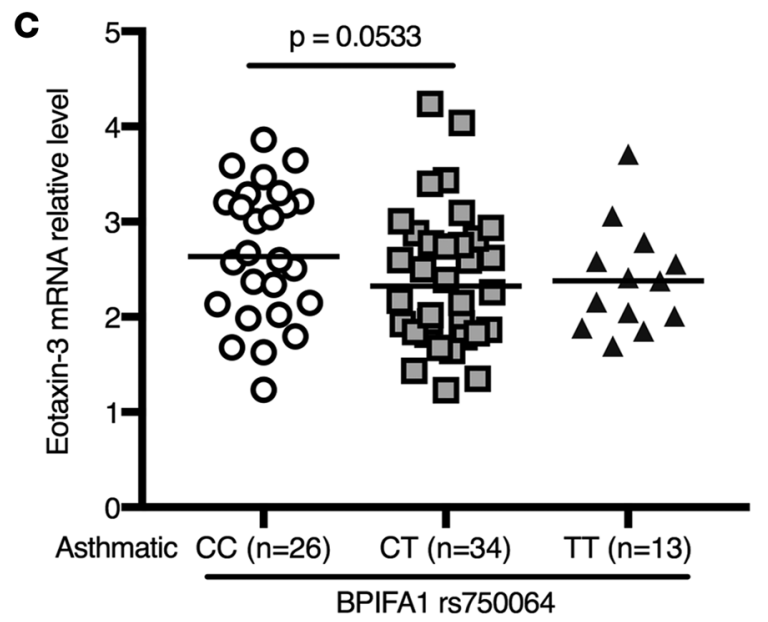

Figure 4. Effect of rs750064 genotypes on type 2 markers. (A) Periostin mRNA expression ( $n=26$ for CC; $n=34$ for CT; and $n=13$ for TT). (B) A negative correlation of BPIFA1 mRNA with periostin mRNA in noncultured nasal brushing cells from 73 subjects with asthma ( $n=48$ adults, $n=25$ children) using a Pearson's correlation analysis. (C) Eotaxin-3 mRNA expression ( $n=26$ for CC; $n=34$ for CT; and $n=13$ for TT). The black line represents the median value, and the Mann-Whitney test was used for $\mathbf{A}$ and $\mathbf{C}$.

In summary, our study has shown a significant impact of genetic variations on BPIFA1 expression in the minimally invasive nasal brushing samples from patients with asthma. The interactions of genetically predetermined BPIFA1 expression within a type 2 airway milieu may form a vicious cycle in worsening airway inflammation. BPIFA1-targeted therapy may offer a novel opportunity to reduce type 2 inflammation in a subset of patients with asthma expressing lower levels of BPIFA1 associated with rs750064.

\section{Methods}

Subject recruitment. Nonsmoking ( $<10$ pack years and no smoking in past year) patients with asthma were recruited in SARP cohorts 1, 2, and 3. The categorization of severe asthma was based on the European Respiratory Society and American Thoracic Society guidelines (30). Briefly, severe asthma was defined as that requiring the use of high-dose inhaled and/or oral corticosteroids, along with a second controller, to keep the asthma controlled or remaining uncontrolled despite therapy. Evaluations included pulmonary function testing, total serum IgE, white blood cell counts and differentials, and FeNO measurements $(31,32)$.

Subjects who did not meet the criteria for severe asthma were divided into mild or moderate asthma on the basis of lung function and inhaled corticosteroid (ICS) use (32). Mild asthma was defined as an FEV1 of greater than $80 \%$ with little to no ICS use (32). Moderate asthma was defined as an FEV1 of less than $80 \%$ with low to moderate ICS use (32). 

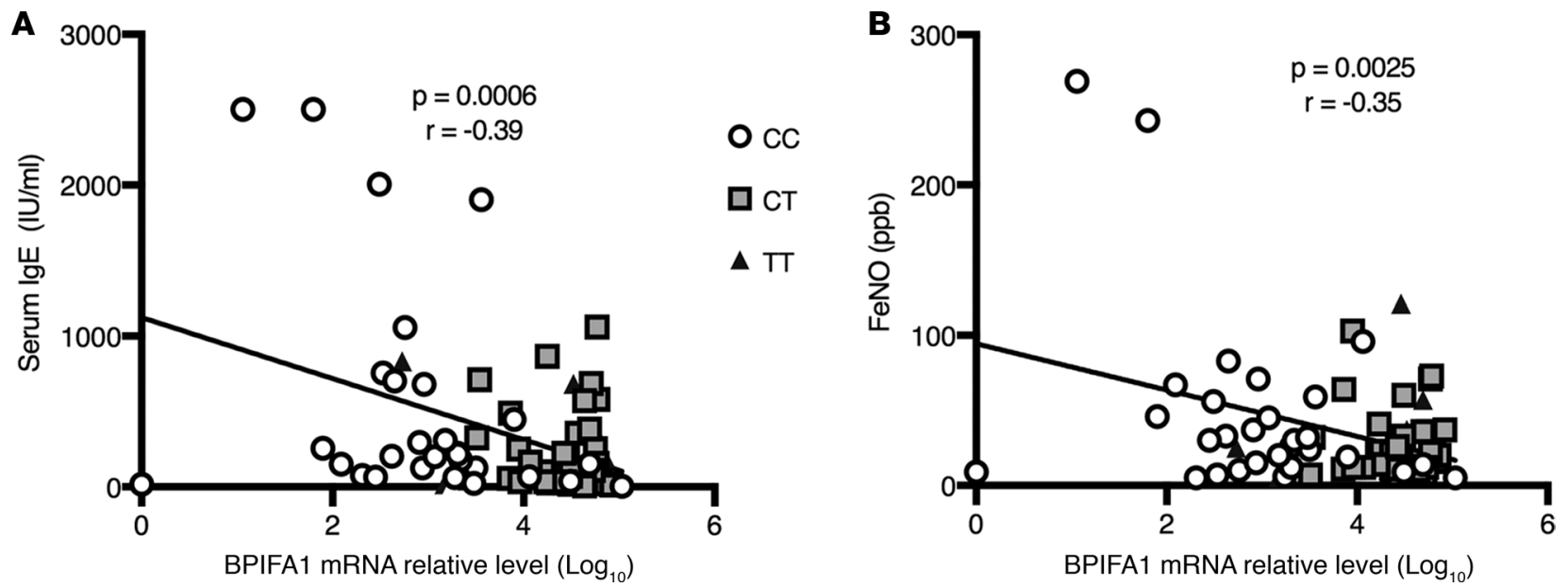

Figure 5. Association of nasal epithelial BPIFA1 mRNA expression with type 2 markers in clinical samples. (A) Correlation of BPIFA1 mRNA levels with serum IgE levels $(n=74)$. (B) Correlation of BPIFA1 mRNA levels with FeNO, parts per billion ( $\mathrm{ppb})$, levels $(n=73)$ in asthmatic subjects with mild to severe disease. A Pearson's correlation analysis was performed.

Nasal brushing samples and clinical outcomes from 74 subjects of the SARP3 cohort were used in this study. SARP3 is the most current cohort, for which 118 pediatric (ages 6 to 17 years old) and 526 adult (18 years and older) subjects with asthma were recruited (33).

To further define the implication of BPIFA1 genetic variations in clinical outcomes, data from 1,016 asthma subjects previously included in the genome-wide association studies (GWAS) from all 3 SARP cohorts were analyzed. Additionally, 123 healthy controls from the SARP1 and SARP2 cohorts, as reported in our previous study (34), were included.

Nasal brush collection. Nasal brushing epithelial cells $(n=74)$ were collected from mild to severe adult $(n=50$, Table 1$)$ and pediatric $(n=24$, Table 2$)$ patients with asthma. Each subject underwent 2 nasal brushings, 1 per nostril. A cytology brush was inserted into the nasal cavity just past the inferior turbinate and then rolled laterally so that the epithelium just under the inferior turbinate was brushed (19). Once collected, 1 nasal brush was put in a collection tube containing freezing media (60\% DMEM, 30\% FBS, 10\% DMSO), which was later used for epithelial cell culture, and the other brush was placed into a collection tube containing Qiagen RLT Plus buffer and 2 M DTT (MilliporeSigma) and then extracted for DNA and RNA extraction using a Qiagen AllPrep DNA/RNA Mini Kit.

Detection of rs750064 SNP alleles. BPIFA1 rs750064 in nasal brushing samples was genotyped by sequencing the region flanking rs750064. Genomic DNA, isolated from noncultured brushed nasal epithelial cells, was first amplified by PCR to generate a 339-bp DNA fragment spanning the SNP region using high-fidelity DNA polymerase and the following specific oligonucleotide primers: 5'-GGGGCCAA-

Table 3. Demographic information of subjects with asthma (non-Hispanic Whites) from the SARP1, SARP2, and SARP3 cohorts

\begin{tabular}{|c|c|c|c|}
\hline rs750064 & CC & CT & TT \\
\hline $\mathrm{N}$ & 237 & 514 & 265 \\
\hline Sex (male/female) & $96 / 141$ & $174 / 340$ & $90 / 175$ \\
\hline Age (yrs) & $38.6 \pm 16.7$ & $39.0 \pm 17.0$ & $40.5 \pm 16.6$ \\
\hline Age of asthma onset (yrs) & $14.9 \pm 14.7$ & $15.9 \pm 16.0$ & $18.1 \pm 15.7$ \\
\hline Asthma severity & 136 mild/moderate, 101 severe & 281 mild/moderate, 232 severe & 147 mild/moderate, 118 severe \\
\hline FEV1/FVC & $0.71 \pm 0.12$ & $0.72 \pm 0.12$ & $0.72 \pm 0.12$ \\
\hline FEV1 (\%) & $76.0 \pm 21.6^{A}$ & $77.4 \pm 21.5$ & $78.8 \pm 21.1$ \\
\hline$\%$ on ICS & 80 & 80 & 79 \\
\hline$\%$ on other CS & 23 & 18 & 20 \\
\hline BMI & $28.8 \pm 7.6$ & $28.9 \pm 7.9$ & $29.3 \pm 8.1$ \\
\hline
\end{tabular}

${ }^{A} P<0.05$. FVC, forced vital capacity; ICS, inhaled corticosteroids; other CS, oral/injected corticosteroids; BMI, body mass index (kg/m²). 
Table 4. Demographic information of 123 healthy controls (non-Hispanic Whites) from SARP1 and SARP2 cohorts

\begin{tabular}{lccc}
\hline rs750064 & cC & CT & TT \\
N & 30 & 68 & 25 \\
Sex & 8 male, 22 female & 26 male, 42 female & 9 male, 16 female \\
Age (yrs) & $30.8 \pm 11.2$ & $32.9 \pm 12.0$ & $31.0 \pm 9.3$ \\
FEV1/FVC & $0.83 \pm 0.07$ & $0.82 \pm 0.07$ & $0.80 \pm 0.07$ \\
FEV1 (\%) & $99.3 \pm 11.2$ & $97.9 \pm 10.8$ & $97.4 \pm 10.3$ \\
FVC, forced vital capacity. & & & \\
\hline
\end{tabular}

GAGATGAGACTG-3' (forward primer) and 5'-CTCACTTCTGCCGAGGAGTC-3' (reverse primer). The resulting DNA fragment was purified by agarose gel electrophoresis and extracted from the gel as a single band using QIAquick Gel Extraction Kit (Qiagen). The purified product was then subjected to Sanger sequencing using the following internal oligonucleotide primer: 5'-TGAGACTGAGGTCCCTTCCT-3'. Reference genomic DNA samples containing either one of the SNP variants were obtained from Coriell Institute for Medical Research (Camden, New Jersey) and were used as controls for each variant. These included reference samples NA07348 (CC genotype), NA10831 (CT genotype), and NA06990 (TT genotype). The allelic frequency within the study population was 0.5946 for the $\mathrm{C}$ allele and 0.4054 for the $\mathrm{T}$ allele. These allelic frequencies are representative of the normal population distribution (35).

BPIFA1 rs750064 in the SARP1, 2, and 3 cohorts was identified with the Illumina Human Hap1M BeadChip (34).

ALI culture of nasal epithelial cells. Nasal epithelial cells were cultured and expanded on irradiated 3T3 fibroblasts (ATCC) in the presence of a Rho kinase inhibitor Y-27632 as described previously (36, 37). Nasal epithelial cells from 22 patients with asthma (10 CC, 8 CT, 4 TT) chosen randomly, 11 to 66 years of age and ranging in severity from mild to severe, were seeded onto collagen-coated, 12-well Transwell plates (Transwell 3460, Corning Incorporated) at $4 \times 10^{4}$ cells/well in medium made up of DMEM and Airway Epithelial Cell Growth Media Kit (PromoCell) (1:1). After 7 days of submerged culture, $100 \%$ confluence was reached, and the cells were shifted to ALI by removing all but $50 \mu 1$ of medium from the apical surface to induce mucociliary differentiation. Cells were allowed to differentiate for 14 days, which was based on our previous work where cilia were enriched after 14 days of ALI $(20,21,38)$. On day 14 of ALI culture, cells were pretreated with $10 \mathrm{ng} / \mathrm{ml}$ of IL-13 (R\&D Systems) and/or $20 \mu \mathrm{g} / \mathrm{ml}$ of rhBPIFA1 protein or BSA (control) for 24 hours. Following the 24-hour pretreatment the cells were again treated with $10 \mathrm{ng} / \mathrm{ml}$ of IL-13 and/or $20 \mu \mathrm{g} / \mathrm{ml}$ of rhBPIFA1 protein. Cells and supernatants were harvested 24 hours later. Briefly, $200 \mu 1$ of Dulbecco's PBS (Thermo Fisher Scientific) was added to the apical surface and incubated at $37^{\circ} \mathrm{C}$ for 10 minutes, after which both apical and basolateral supernatants were collected. Our group produced and purified rhBPIFA1 protein as described previously (16).

Enzyme-linked immunosorbent assay. BPIFA1 protein levels in the apical supernatant were measured using a direct BPIFA1 ELISA that we developed (14). Eotaxin-3 protein levels from the basolateral supernatants were measured using a DuoSet ELISA kit from R\&D Systems.

Quantitative real-time PCR. RNA from noncultured or cultured nasal epithelial cells was extracted using the RNeasy Mini Kit (Qiagen). We performed reverse transcription on 500 ng of RNA to produce cDNA using the Bio-Rad T100 thermal cycler.

TaqMan gene expression assay for human eotaxin-3 and periostin was obtained from Thermo Fisher Scientific, and a custom-made gene expression assay for human BPIFA1 was obtained from Integrated DNA Technologies (14). The housekeeping gene 18S rRNA was used as an internal control to normalize the expression of target genes. The comparative cycle of threshold $(\Delta \Delta \mathrm{Ct})$ method was used to demonstrate the relative levels of the target genes.

Statistics. One-way ANOVA followed by a Tukey's post hoc test was used for multiple comparisons of normally distributed data. Two-tailed Student's $t$ test was used when only 2 groups were compared. For nonparametric data, the Mann-Whitney test was used when only 2 groups were compared, and the Kruskal-Wallis test was applied for multiple comparisons. Pearson's correlation analysis was performed to determine the relationship of various parameters as reported in the Results section. A linear regression 
additive genetic model with adjustment of age, sex, and race (34) was used to determine the association of rs750064 with BPIFA1 expression and pulmonary function (e.g., FEV1\%). A $P$ value less than 0.05 was considered significant.

Study approval. Research subjects were recruited at 5 SARP centers. Each center had the IRB approval to enroll the subjects with asthma into the SARP cohort and perform the nasal brushings. All the adult subjects or the parents/guardians of the children provided written informed consent (32).

\section{Author contributions}

NS, SG, and NP conducted experiments and were responsible for the acquisition of data. AD and NS conducted the SNP genotyping. NS and RB expanded the nasal epithelial cells. MAS optimized the nasal epithelial cell expansion and cell culture protocol. NJN, LCD, MC, AMC, WGT, and JB provided the nasal epithelial cells. XL, ERB, and DAM provided and analyzed BPIFA1 SNP and clinical data in the large SARP cohort. JF, BRP, and DTM provided clinical data for the SARP3 research subjects. WCM is the principal investigator of Wake Forest. GAH directed the genotyping and GWAS at Wake Forest. NS, XL, YPD, SEW, and HWC designed the research studies, analyzed the experimental and clinical results, designed the original hypothesis, and all contributed to the writing of the final manuscript. All authors were involved in the editing of the manuscript.

\section{Acknowledgments}

The authors thank Jamie Everman and Cydney Rios at National Jewish Health for their help on nasal epithelial cell processing and culture, Courtney Moreno at UCSF and Gina Crisafi at University of WisconsinMadison for their help in subject recruitment, and Seo Y. Park at the University of Pittsburgh for statistical analysis. Funding sources: NIH/NHLBI R01HL125128, U10HL109257, UL1TR00448, and U10HL109168.

Address correspondence to: Y. Peter Di or Sally E. Wenzel, Department of Environmental and Occupational Health, University of Pittsburgh, 130 De Soto St., Pittsburgh, Pennsylvania 15261, USA. Phone: 412.624.8718; Email: peterdi@pitt.edu (YPD). Phone: 412.802.6859; Email: swenzel@pitt.edu (SEW). Or to: Hong Wei Chu, Department of Medicine, National Jewish Health, 1400 Jackson St., Room A639, Denver, Colorado 80206, USA. Phone: 303.398.1689; Email: chuhw@njhealth.org.

1. Fahy JV. Type 2 inflammation in asthma — present in most, absent in many. Nat Rev Immunol. 2015;15(1):57-65.

2. Dunican EM, Fahy JV. The Role of Type 2 Inflammation in the Pathogenesis of Asthma Exacerbations. Ann Am Thorac Soc 2015;12(suppl 2):S144-S149.

3. Robinson D, et al. Revisiting Type 2-high and Type 2-low airway inflammation in asthma: current knowledge and therapeutic implications. Clin Exp Allergy. 2017;47(2):161-175.

4. Bingle CD, Bingle L. Characterisation of the human plunc gene, a gene product with an upper airways and nasopharyngeal restricted expression pattern. Biochim Biophys Acta. 2000;1493(3):363-367.

5. Campos MA, Abreu AR, Nlend MC, Cobas MA, Conner GE, Whitney PL. Purification and characterization of PLUNC from human tracheobronchial secretions. Am J Respir Cell Mol Biol. 2004;30(2):184-192.

6. Bingle CD, Craven CJ. PLUNC: a novel family of candidate host defence proteins expressed in the upper airways and nasopharynx. Hum Mol Genet. 2002;11(8):937-943.

7. Wang S, Li WL, Lü LC, Yao KT. [Expressions of short palate, lung and nasal epithelium clone 1 in different human tissues]. Nan Fang Yi Ke Da Xue Xue Bao. 2016;36(5):617-621.

8. Leclair EE. Four BPI (bactericidal/permeability-increasing protein)-like genes expressed in the mouse nasal, oral, airway and digestive epithelia. Biochem Soc Trans. 2003;31(pt 4):801-805.

9. Duke BO, Moore PJ, Anderson J. Studies on factors influencing the transmission of onchocerciasis. VII. A comparison of the Onchocerca volvulus transmission potentials of Simulium damnosum populations in four Cameroon rain-forest villages and the pattern of onchocerciasis associated therewith. Ann Trop Med Parasitol. 1972;66(2):219-234.

10. Sayeed S, Nistico L, St Croix C, Di YP. Multifunctional role of human SPLUNC1 in Pseudomonas aeruginosa infection. Infect Immun. 2013;81(1):285-291.

11. Gally F, et al. SPLUNC1 promotes lung innate defense against Mycoplasma pneumoniae infection in mice. Am J Pathol. 2011;178(5):2159-2167.

12. Lukinskiene L, et al. Antimicrobial activity of PLUNC protects against Pseudomonas aeruginosa infection. J Immunol. 2011;187(1):382-390.

13. Akram KM, et al. An innate defense peptide BPIFA1/SPLUNC1 restricts influenza A virus infection. Mucosal Immunol. 2018;11(1):71-81.

14. Chu HW, et al. Function and regulation of SPLUNC1 protein in Mycoplasma infection and allergic inflammation. J Immunol. 2007;179(6):3995-4002.

15. Gross CA, Bowler RP, Green RM, Weinberger AR, Schnell C, Chu HW. $\beta 2$-Agonists promote host defense against bacterial 
infection in primary human bronchial epithelial cells. BMC Pulm Med. 2010;10:30.

16. Thaikoottathil JV, et al. SPLUNC1 deficiency enhances airway eosinophilic inflammation in mice. Am J Respir Cell Mol Biol. 2012;47(2):253-260

17. He Y, et al. Association of PLUNC gene polymorphisms with susceptibility to nasopharyngeal carcinoma in a Chinese population. J Med Genet. 2005;42(2):172-176.

18. Saferali A, et al. Polymorphisms associated with expression of BPIFA1/BPIFB1 and lung disease severity in cystic fibrosis. Am J Respir Cell Mol Biol. 2015;53(5):607-614.

19. Poole A, et al. Dissecting childhood asthma with nasal transcriptomics distinguishes subphenotypes of disease. J Allergy Clin Immunol. 2014;133(3):670-8.e12.

20. Ito Y, et al. IL-13 induces periostin and eotaxin expression in human primary alveolar epithelial cells: Comparison with paired airway epithelial cells. PLoS One. 2018;13(4):e0196256.

21. Roberts N, Al Mubarak R, Francisco D, Kraft M, Chu HW. Comparison of paired human nasal and bronchial airway epithelial cell responses to rhinovirus infection and IL-13 treatment. Clin Transl Med. 2018;7(1):13.

22. Coleman JM, et al. Epithelial eotaxin-2 and eotaxin-3 expression: relation to asthma severity, luminal eosinophilia and age at onset. Thorax. 2012;67(12):1061-1066.

23. Woodruff PG, et al. Genome-wide profiling identifies epithelial cell genes associated with asthma and with treatment response to corticosteroids. Proc Natl Acad Sci U S A. 2007;104(40):15858-15863.

24. Zhang K, et al. Decreased epithelial and sputum miR-221-3p associates with airway eosinophilic inflammation and CXCL17 expression in asthma. Am J Physiol Lung Cell Mol Physiol. 2018;315(2):L253-L264.

25. Wei Y, et al. The antimicrobial protein short palate, lung, and nasal epithelium clone 1 (SPLUNC1) is differentially modulated in eosinophilic and noneosinophilic chronic rhinosinusitis with nasal polyps. J Allergy Clin Immunol. 2014;133(2):420-428.

26. Yeh TH, Lee SY, Hsu WC. Expression of SPLUNC1 protein in nasal polyp epithelial cells in air-liquid interface culture treated with IL-13. Am J Rhinol Allergy. 2010;24(1):17-20.

27. Chu HW, et al. Transforming growth factor- $\beta 2$ induces bronchial epithelial mucin expression in asthma. Am J Pathol. 2004;165(4):1097-1106.

28. Liu J, et al. Role of IL-13R $\alpha 2$ in modulating IL-13-induced MUC5AC and ciliary changes in healthy and CRSwNP mucosa. Allergy. 2018;73(8):1673-1685

29. Woodruff PG, et al. T-helper type 2-driven inflammation defines major subphenotypes of asthma. Am J Respir Crit Care Med. 2009;180(5):388-395.

30. Chung KF, et al. International ERS/ATS guidelines on definition, evaluation and treatment of severe asthma. Eur Respir J. 2014;43(2):343-373.

31. Miller MR, et al. Standardisation of spirometry. Eur Respir J. 2005;26(2):319-338.

32. Moore WC, et al. Characterization of the severe asthma phenotype by the National Heart, Lung, and Blood Institute's Severe Asthma Research Program. J Allergy Clin Immunol. 2007;119(2):405-413.

33. Teague WG, et al. Baseline features of the severe asthma research program (SARP III) cohort: differences with age. $J$ Allergy Clin Immunol Pract. 2018;6(2):545-554.e4.

34. Li X, et al. Genome-wide association studies of asthma indicate opposite immunopathogenesis direction from autoimmune diseases. J Allergy Clin Immunol. 2012;130(4):861-8.e7.

35. O'Leary NA, et al. Reference sequence (RefSeq) database at NCBI: current status, taxonomic expansion, and functional annotation. Nucleic Acids Res. 2016;44(D1):D733-D745.

36. Chu HW, et al. CRISPR-Cas9-mediated gene knockout in primary human airway epithelial cells reveals a proinflammatory role for MUC18. Gene Ther. 2015;22(10):822-829.

37. Suprynowicz FA, et al. Conditionally reprogrammed cells represent a stem-like state of adult epithelial cells. Proc Natl Acad Sci US A. 2012;109(49):20035-20040

38. Jiang D, Schaefer N, Chu HW. Air-Liquid Interface Culture of Human and Mouse Airway Epithelial Cells. Methods Mol Biol. 2018;1809:91-109. 